\title{
DÜBLIN
}

Technological University Dublin

ARROW@TU Dublin

2005-01-01

\section{Interaction of Single Walled Carbon Nanotubes with Starch-based Systems}

\author{
Alan Casey \\ Technological University Dublin, alan.casey@tudublin.ie \\ Gerald Farrell \\ Technological University Dublin, gerald.farrell@tudublin.ie \\ Mary McNamara \\ Technological University Dublin, Mary.McNamara@tudublin.ie
}

See next page for additional authors

Follow this and additional works at: https://arrow.tudublin.ie/radcon

Part of the Physics Commons

\section{Recommended Citation \\ This Conference Paper is brought to you for free and open access by the Radiation and Environmental Science Centre at ARROW@TU Dublin. It has been accepted for inclusion in Conference papers by an authorized administrator of ARROW@TU Dublin. For more information, please contact arrow.admin@tudublin.ie, aisling.coyne@tudublin.ie, gerard.connolly@tudublin.ie.}

Casey, A., Farrell, G.F. \& McNamara, M. (2005). Interaction of single walled carbon nanotubes with starchbased systems. Proceedings of SPIE, vol. 5824, pg. 182-189. doi:10.1117/12.604329 


\section{Authors}

Alan Casey, Gerald Farrell, Mary McNamara, Hugh Byrne, and Gordon Chambers

This conference paper is available at ARROW@TU Dublin: https://arrow.tudublin.ie/radcon/2 


\title{
Interaction of Single Walled Carbon Nanotubes with Starch-based Systems
}

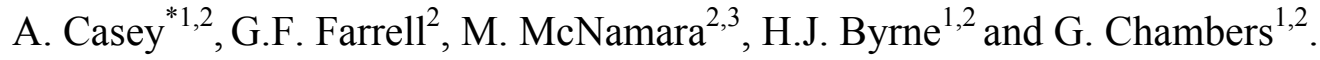 \\ ${ }^{1}$ School of Physics, Dublin Institute of Technology, Kevin Street, Dublin 08, Ireland. \\ ${ }^{2}$ FOCAS, Dublin Institute of Technology, Kevin Street, Dublin 08, Ireland. \\ ${ }^{3}$ School of Chemistry, Dublin Institute of Technology, Kevin Street, Dublin 08, Ireland.
}

\begin{abstract}
The interaction of carbon nanotubes with soft organic molecules such as cyclodextrins and other saccharides has recently been shown to produce water-soluble composites. Such systems offer considerable advantages over polymer based composites due to their biocompatibility and noncovalent coupling which can potentially preserve the unique properties of the tubes. The mechanism of interaction of such systems has been proposed to be dominated by hydrophobic and hydrophilic interactions along the surface of the tube. In this study a number of composite systems have been formed with HiPco carbon nanotubes using starch and starch based polymers. The first composite formed with starch was characterised using a range of spectroscopic, imaging and thermal techniques which showed clear evidence of an intermolecular interaction. The second and third composite systems formed utilised biodegradable starch based polymers namely starch cellulose acetate (SCA) and starch polycrapolactone (SPCL). The characterisation of these systems will be presented. Finally the potential for enzyme immobilisation into the composite matrix was investigated.
\end{abstract}

Keywords: Nanotubes, Starch, Raman, Infra-Red, Electronic, Spectroscopy.

\section{INTRODUCTION}

The interaction of carbon nanotubes with soft organic molecules has begun to emerge as an accepted route towards the purification and isolation of this unique fullerene based material. While experiments performed using polymeric and surfactant based routes have shown considerable efficiencies for the purification and dispersion of single walled carbon nanotubes [1]. Much of the work has focused on using harsh solvent based systems with poor affinities for biological systems. The report by Bandyopadhyaya et al [2] that gum Arabic, a polysaccharide, could form stable well dispersed suspensions of SWNT in aqueous solution excited the entire area with the potential that such composite systems could hold the key to the successful integration of nanotubes into biological environments.

The interaction of saccharides with fullerenes was demonstrated as early as 1993 when a $\mathrm{C}_{60}-\gamma$ cyclodextrin complex was formed [3]. It has since been suggested that these oligosaccharides interact with the SWNT via a Van der Waals interaction. Furthermore it is proposed that these soft organic materials can actually cut the nanotube although no specific mechanism was proposed. It was however shown spectroscopically that the interaction was along the length of the tube and appeared to introduce a compressive strain on the tube surface consistent with a plausible cutting effect [4]. Most recently amylose a polymeric form of glucose and a constituent of starch has been proposed to weakly interact with HCNT via non-specific hydrophobic interactions [5].

Although spectroscopic evidence for such interactions was weak, thermal analysis and microscopy did indicated the formation of a starch-nanotube complex. In this study further spectroscopic analysis is carried out on a similar starch system to confirm its potential as a dispersing agent for carbon nanotubes. Work carried out by Star et al [6] proposed an enhanced interaction of amylose and HCNT by creating an inclusion complex of amylose and excess iodine prior to the addition of the HCNT creating a coiled starch-nanotube complex. This study investigates the spectroscopic effects of complex formation of starch with nanotubes.

\section{EXPERIMENTAL}

In this Study HCNT were used to form three complexes with starch. The first simple complex of starch and nanotubes was created by dispersing HCNT $(1 \mathrm{mg})$ in $25 \mathrm{ml}$ of a starch solution $(10 \mathrm{~g} / \mathrm{l})$ followed by ultrasonication $(4$ minutes medium power). 
The second complex also utilised the widely known starch iodine complex. Starch in solution naturally resides in a helical arrangement, upon the addition of iodine an inclusion complex is formed where by the iodine resides inside the helical starch giving rise to a characteristic blue black coloured solution. A solution of the starch (10g/l) was saturated with iodine yielding the characteristic blue black coloured starch iodine inclusion complex, this solution was then allowed to rest for 24 hours to allow any un-reacted iodine to precipitate and the supernatant was then decanted and used to form the second starch nanotube complex. $1 \mathrm{mg}$ of HCNT were then dispersed in $25 \mathrm{ml}$ of in the supernatant. The nanotube containing solution then under went a process of sonication ( 4 minutes medium power in a sonic bath) and centrifugation (15 minutes at 3000RPM), this process resulted in a clear aqueous solution of starch and HCNT.

The third complex again used starch, but here the iodine was replaced with bromine, which again resulted in an inclusion complex of the starch being formed prior to the addition of the HCNT. The bromine-starch solution was again allowed to settle before the supernatant was decanted off thereby limiting any potential effects of excess or un-reacted bromine. This solution was then used to form the nanotube complex. Upon the addition of the nanotube the solution under went a process involving sonication and centrifugation as had been carried out on the iodine based complex.

It should be noted that the first simple system of starch and HCNT was essentially used as a control as a spectroscopic study has been previously reported by Cheng-yi Lii et al [ref] to investigate the effects of the iodine and the bromine on the spectroscopic characteristics of the starch HCNT complex. All complexes were then characterised using a variety of spectroscopic techniques. Vibrational analysis was carried out using an Instruments S.A LabRam 1B Raman microscope and a Perkin Elmer Spectrum GX. Electronic analysis was carried out with the use of a Perkin Elmer Lamada 900 UV/VIS/NIR spectrometer. Circular dicroism measurements were carried out using a Jasco J-810 spectropolarimeter.

\section{RESULTS}

Initially it was visually noted that the different methods resulted in the formation of a water-soluble complex of differing colour. The first complex of amylose and HCNT resulted in the formation of a clear solution, which held the HCNT in suspension for approximately three weeks. It is postulated that amylose, a linear polymer of 1-4 linked $\alpha$-D glucose units which resides in a left handed helical arrangement in an aqueous environment, holds the HCNT in suspension by hydrophobic-hydrophilic interactions with the nanotube as has been previously reported [5].

The second complex which utilised a starch iodine inclusion complex to form a more ordered helical arrangement of amylose by inclusion prior to the addition of the HCNT, again resulted in a clear aqueous solution of nanotubes, as has been previously reported, it is postulated that this colour change from blue black to clear is an indication that the iodine within the helical starch has been completely displaced by the nanotube, resulting in a starch wrapped nanotube complex. The resultant complex permitted the HCNT to remain in solution for up to six weeks.

The third complex formed with starch uses bromine to form a more ordered helical arrangement of amylose by inclusion prior to the addition of the HCNT. The resultant complex permitted the HCNT to remain in solution for up to six weeks and retained the characteristic orange colour of the bromine amylose inclusion complex. This is in stark contrast to the complex formed with amylose alone and the complex formed by Star et al [6] (which used iodine as opposed to bromine). Both of these resulted in clear colourless solutions. It was proposed by Star et al [6] that the iodine is completely displaced by the inclusion of the nanotubes into the cavity of the starch thereby rendering the solution colourless. As a result the colour retention of the complex here may be an indication that all the bromine is not displaced but rather due to its smaller size can be readily accommodated in the cavity with the nanotubes forming a tertiary complex or composite. Of course the possibility that the colour may the result of excess bromine cannot be ruled out. However the volume of bromine used to produce the initial starch inclusion complex in successive runs was significantly reduced, yet the orange colour was still retained upon the addition of the tubes.

Due to the nature of the complexes produced (differing colour) electronic spectroscopic analysis was the first technique employed to verify the presence of HCNT in the samples. The absorption spectrum of HCNT (Fig.1a) consists of three 
broad absorption peaks centred approximately at $1200 \mathrm{~nm}, 800 \mathrm{~nm}$ and $270 \mathrm{~nm}$. The peaks at $1200 \mathrm{nmn}$ and $800 \mathrm{~nm}$ being attributed to optical transitions between mirror image spikes in the density of states, with the feature at $270 \mathrm{~nm}$ attributed to the $\pi$-plasma frequency of $5 \mathrm{eV}$ in carbon materials [7], Close examination of the absorption spectra of the first two complexes (i.e. formed with starch alone and with the starch iodine inclusion) did not show any spectral evidence of the presence of HCNT in the sample nor of any complex formation. However in the complex formed with bromine and starch HCNT features were present in the complexes spectra verifying the presence of HCNT. Using the broad peak in the NIR region (Fig.1.d) the diameter distribution [8] of the HCNT used was estimated to be $0.8 \mathrm{~nm}-1.36 \mathrm{~nm}$. In comparison, the absorption spectrum of the starch Figure 1 (c) appears to be relatively featureless and a similar spectrum to this was obtained for the starch nanotube complex. Figure 1 (b) shows the absorption spectrum of the starch-bromineHCNT complex. It can be seen there are HCNT features present in the NIR region which indicates some degree of diameter selectivity towards the mid to lower range of the tubes present in the pristine sample. However the spectra do not contain any definitive evidence of the formation of a true chemical complex but rather indicates the presence of HCNT in the sample.
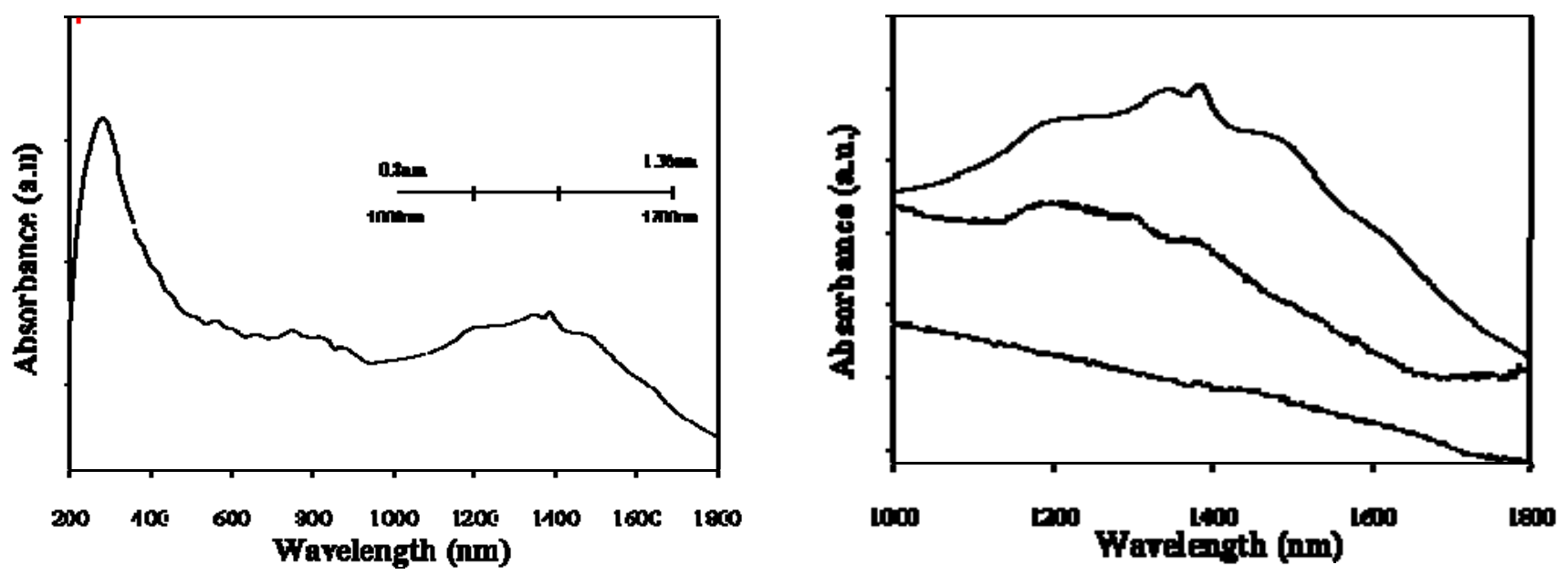

Figure 1 UV-Vis-NIR Absorption Spectra (a) HiPco CNT (b) Close up of NIR Region (I) Raw HiPco (II) Starch Bromine HCNT Complex (III) Starch

Further electronic analysis was sought using Spectropolarimetry. In Spectropolarimetry there are two types of measurement linear and circular dicroism, in this study Circular Dicroism (CD) was employed to further probe the electronic properties of the complexes. There are two main conditions necessary for a substance to produce a circular dicroism signal firstly the molecule must be chiral and secondly there must be an absorbance band present as the CD signal is essentially the difference in absorption between left and right polarised light. CD has been widely used to gain information from inclusion complexes [ref]. However the exact nature of such complexes is often difficult to ascertain for electronic spectroscopic techniques.

Figure 2 shows the CD spectrum obtained for the starch iodine nanotube complex (fig. 2b) and that of starch (fig. 2a). Firstly examination of the starch proved featureless, the starch absorbance spectrum is relatively featureless in the region of interest consisting predominately of a large background scatter with literature indicating its absorbance features occurring in the far UV typically below (180nm) so the lack of a CD signal in the region studied was as expected. However upon the examination of the complex spectrum there is the appearance of a negative CD feature below $205 \mathrm{~nm}$. As neither starch nor nanotubes have an absorbance band in this region. The appearance of this negative feature is unexplained. There are a number of possible origins for this feature firstly it may be a starch feature shifted upwards due to an inclusion complex with the nanotubes and indeed similar shifts have been reported for inclusion complexes. Secondly the feature may be a result of the nanotube absorbance at $270 \mathrm{~nm}$ attributed to the $\pi-$ plasma frequency of $5 \mathrm{eV}$ in carbon materials shifted downwards or finally it may be an entirely new feature caused by the complex of starch and nanotubes. 
Figure 2c shows the CD spectrum obtained for the starch bromine nanotube complex, it can be clearly seen that there are two dominate features present centred between $195 \mathrm{~nm}$ and $205 \mathrm{~nm}$. As previously discussed in order for there to be a CD signal there must be an absorbance band present, as effectively CD is the difference between the absorbance of left and right polarised light. This fact makes the exact origin of these two features difficult to assign as was the case in the starch iodine nanotube complex feature. As mentioned earlier nanotubes have an intrinsic feature at $270 \mathrm{~nm}$ attributed to the $\pi-$ plasma frequency of $5 \mathrm{eV}$ in carbon materials so here again the origin of these features may be a result of this downshifted due to complex formation with the starch or an up shift in the starch features or two new composite features

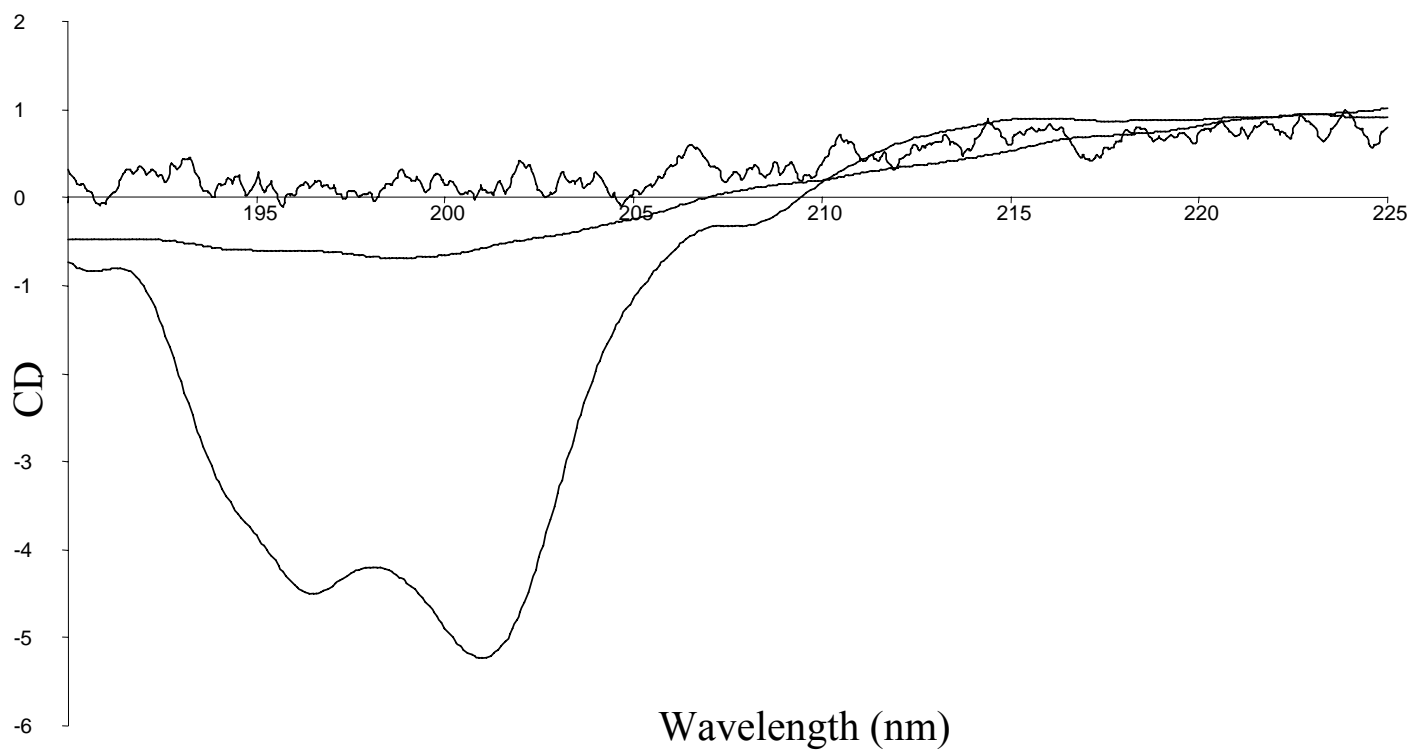

Figure 2. Circular Dicroism Spectra For (a) Starch, (b) Starch iodine Nanotube Complex (c) Starch Bromine Nanotube Complex

So in summary it is postulated that the origin of these features in the CD spectrum could be attributed to a number a factors. Firstly it may originate from the $\alpha-1-4$ Helix of starch, being shifted due to the formation of an inclusion complex with the nanotubes and indeed such shifts in CD peaks are often used as an indication of the degree of inclusion [ref]. But it must also be considered that these features originate from the nanotubes themselves or they may indeed truly new features from the complex. In general the results obtained from electronic spectroscopy showed little evidence to support the formation of a complex. As a result vibrational analysis was carried out to further elucidate the nature of the complex.

Infrared absorption spectroscopy was carried out on all samples to probe their vibrational character. The FTIR spectrum of carbon nanotubes agreed with literature [ref] consisting of three main features at $1600 \mathrm{~cm}^{-1}, 1400 \mathrm{~cm}^{-1}$ and $1180 \mathrm{~cm}^{-1}$ which have been attributed to the $\mathrm{C}-\mathrm{C}$ and $\mathrm{C}=\mathrm{C}$ stretching along the hexagonal array of the nanotube backbone [10]. The infrared signal of starch (Fig..) in contrast is extremely rich and contains a number of strong features primarily due to contributions from $\mathrm{O}-\mathrm{H}, \mathrm{C}-\mathrm{H}$ C-C and C-O stretching. FTIR analysis of the all complexes, showed mainly strong features of starch resulting from glucose with the exception of two new features at $1182 \mathrm{~cm}^{-1}$ and $1509 \mathrm{~cm}^{-1}$ in the bromine, starch, HCNT complex. The feature at $1182 \mathrm{~cm}^{-1}$ could be attributed to the $\mathrm{C}-\mathrm{C}$ stretching in the nanotube. This again indicates that HCNT were present but gave no clear insight into the formation of a complex. The exact origin of the second feature at $1509 \mathrm{~cm}^{-1}$ is still unclear. However it is postulated that this feature may in fact be a previously Raman active IR inactive mode of the HCNT becoming IR active due to a relaxation of the selection rules. For this reason Raman spectroscopy, a technique known to be extremely sensitive to changes in the local environment of the carbon nanotubes was employed to further characterise the complexes. 


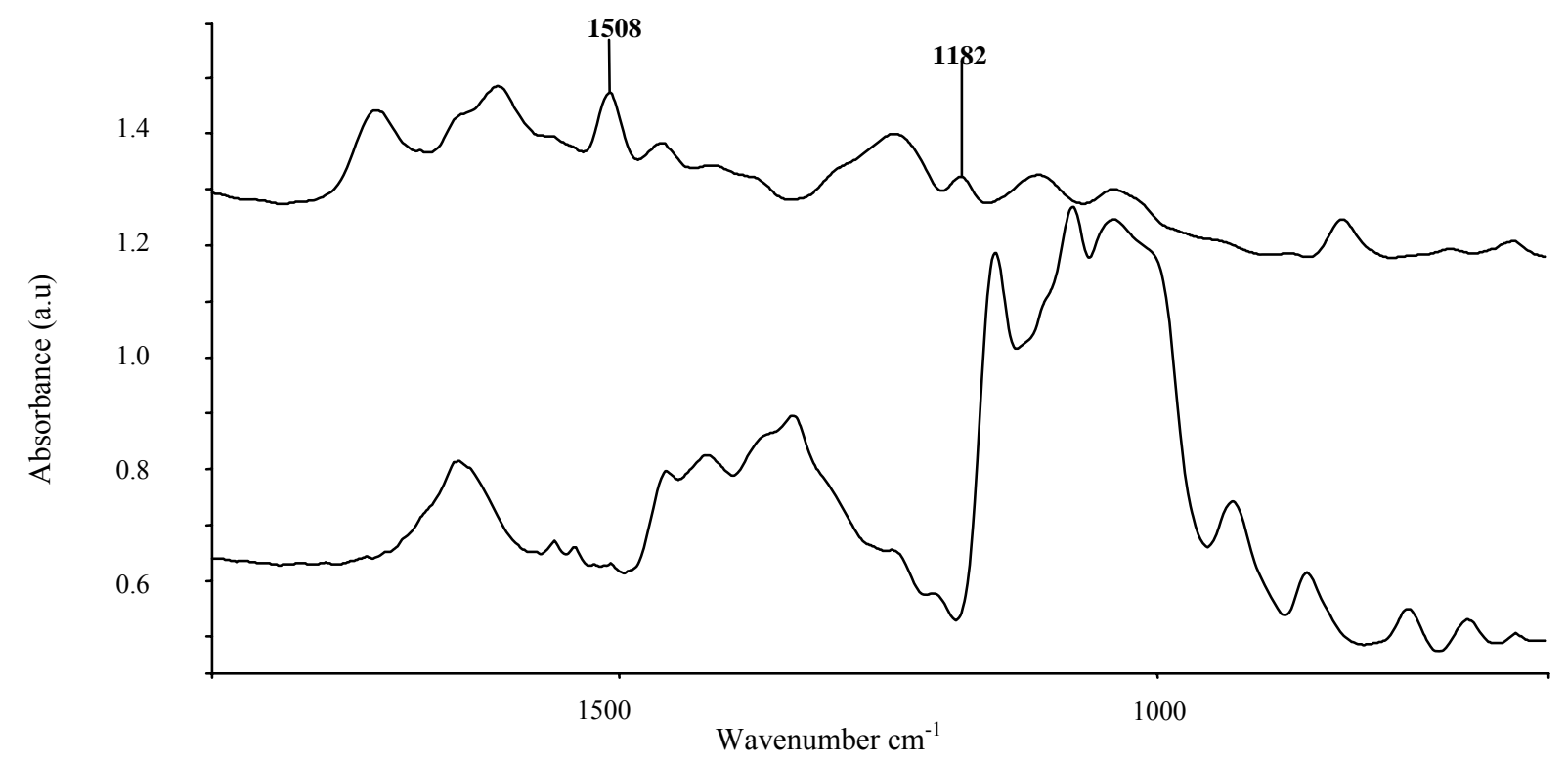

Figure 3. Infra Red Spectra for (a) Starch (b) Starch Bromine Nanotube Complex.

Raman analysis was carried out on all samples at $514 \mathrm{~nm}$ laser excitation. The Raman spectrum of pristine nanotubes (Fig.) consists of three main features, the radial breathing mode, the D line and the $G$ line. These modes are very sensitive to any perturbation to the local environment of the nanotube and are therefore give good indication of complex formation. In the complex formed with starch alone, there was no significant differences in the spectrum, all modes being present and their shapes and positions characteristic of that of the raw nanotubes, as had been previously reported $[5]$.

Examination of the complex formed with starch and iodine the spectrum under goes changes; however with closer examination the spectrum is a direct summation of the starch and nanotube signals with no new composite features present. This type of composite spectra would suggest a very weak interaction giving weight to the notion that the nanotubes are held in suspension by hydrophobic hydrophilic interactions between the starch and the tubes.

This was not the case with the complex involving bromine as a precursor. As mentioned earlier all bromine was not removed in the formation of the complex as indicated by its final colour. For this reason it was postulated that a tertiary composite could have been formed of starch, bromine and HCNT. Supportive evidence for this tertiary system can be seen in the Raman spectrum of the composite (Fig..) by the appearance of four new features in the spectrum at $652.8 \mathrm{~cm}^{-}$ $1,1042.2 \mathrm{~cm}^{-1}, 1658.4 \mathrm{~cm}^{-1}$ and $1726.4 \mathrm{~cm}^{-1}$. It is important to note that this spectrum is not a direct summation of the amylose and HCNT spectra. The new feature present at $1042 \mathrm{~cm}^{-1}$ may be due to IR active modes of the starch becoming Raman active due to changes in symmetry relaxing the normal selection rules.

Indeed close examination of the IR spectrum reveals a number of candidate FTIR modes, which could become Raman active in this region namely the starch IR modes at $1078 \mathrm{~cm}-1$ and $1021 \mathrm{~cm}-1$ which are attributed to C-O stretching and $\mathrm{CH}$ bending respectively. The other three features however appear to be truly new features and while their exact origin is still unknown, their presence undoubtedly indicates the formation of a new starch-bromine-Nanotube complex. Another noticeable feature of this spectrum is the narrowing of the $\mathrm{G}$ mode with a shift in peak position from $1588 \mathrm{~cm}^{-1}$ in the raw sample to $1601 \mathrm{~cm}^{-1}$ in the complex. Similar shifts in this mode have been reported within nanotube saccharide systems such as cyclodextrins [ref], these shifts were attributed to a compressive strain induced by the cyclodextrins absorbing onto the surface of the tube. So the possibility that the starch is having a similar effect on the tubes cannot be ruled out 


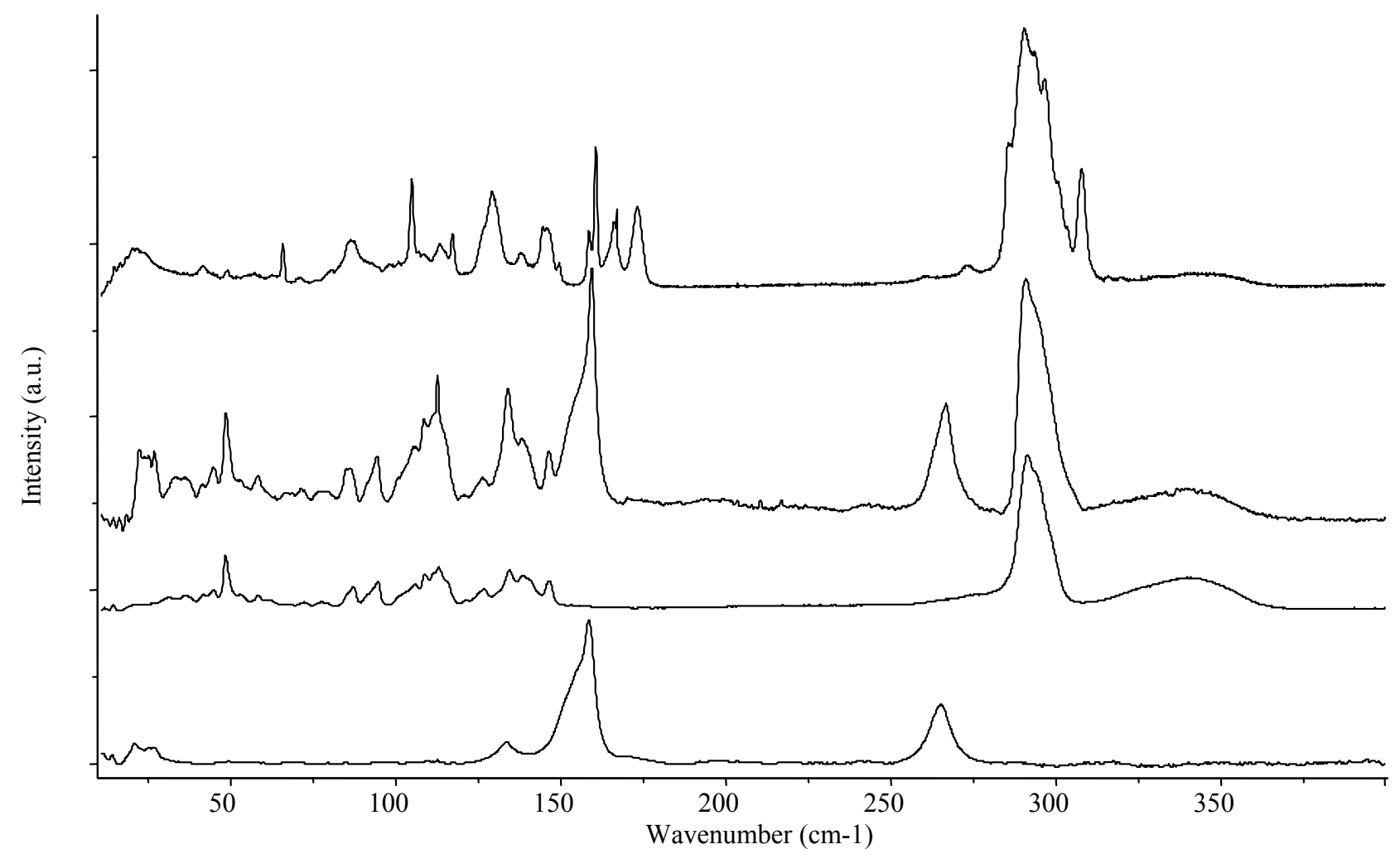

Figure 4. Raman Signal at 514nm excitation of (a) Raw HiPco Nanotubes (b) Starch (c) Starch Iodine Nanotube Complex (d) Starch Bromine Nanotube Complex.

Finally there are also noticeable differences in the radial breathing mode region of the spectra, the RBMs of pristine nanotubes are inversely related to the diameters of the nanotubes [11] and using this relationship, a diameter range for the raw sample was calculated [11] to be from $0.8 \mathrm{~nm}-1.4 \mathrm{~nm}$. This was not the case in the comples formed involving iodine and bromine (Fig.). In this spectral region starch is clear of features (Fig.), facilitating the diameter range of the complex to be calculated directly in a similar fashion to the raw sample. Upon analysis of the starch iodine nanotube complex the calculated range had decreased to $1.00-1.28 \mathrm{~nm}$. Similarly the calculated range of the bromine complex had decreased to $1.00 \mathrm{~nm}-1.25 \mathrm{~nm}$, which is in broad agreement with the degree of selectivity noted from the absorption spectra. Indicating that there is a diameter selectivity towards the mid diameter range of the bulk samples.

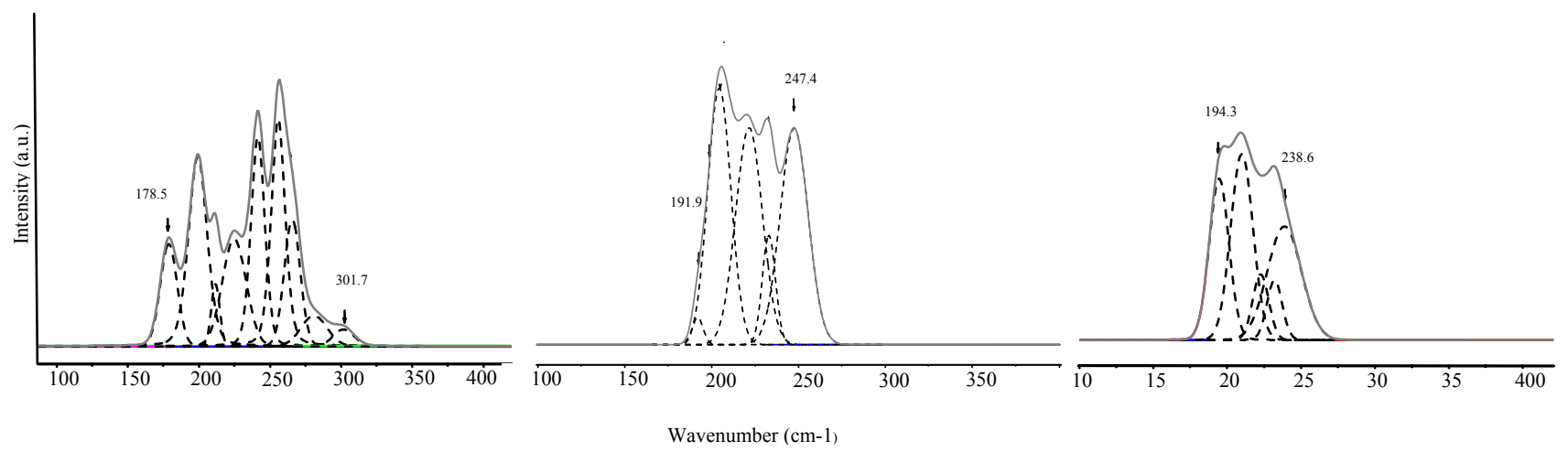

Figure 5. Raman Radial Breathing Mode Region at 514nm excitation of (a) Raw HiPco (b) Starch Iodine Nanotube Complex (c) Starch Bromine Nanotube Complex. 
In summary, the efficiency of starch to act a dispersion agent of nanotubes in an aqueous solution was increased by the addition of iodine and bromine into the system prior to the nanotubes as it facilitated them to remain in suspension for longer in comparison to the complex formed with starch alone. Although electronic analysis showed little evidence to support the notion of a true composite system between the starch and the nanotubes it did verify their presence within the samples by the occurrence of nanotube features in the NIR spectrum for the starch bromine based complex. Further evidence of an interaction was received form circular dicroism studies, although interpretation of these spectra is difficult as the technique is not widely utilised in the characterisation of nanotube systems, changes in the circular dicroism spectra do indicate an effect of the addition of nanotubes to the starch. Vibrational analysis showed clear evidence of an interaction in both the iodine and bromine starch based systems. In particular in the bromine based system supportive evidence for the notion of the formation of a true chemical tertiary complex of bromine starch and nanotubes was provided by the appearance of the new features in the spectrum in both the Raman and IR.

\section{CONCLUSIONS}

It has been shown spectroscopically that a tertiary composite system was created utilising a guest host mechanism of starch. Vibrational spectroscopy showed clear evidence of the formation of complex between the starch and the carbon nanotubes while evidence from the electronic spectroscopy was considerable weaker in it still provided clear signs of an intermolecular interaction. The degree of selectivity of these complexes is still the subject of much speculation and work is ongoing to determine the nature and extent of the interaction. Undoubtedly such composite systems do provide a potential route towards the bio functionalisation and integration of nanotubes into biological environments. However questions are raised about the insufficient displacement of bromine in the final complex

\section{REFERENCES}

